\title{
INCORPORATING UPFC MODEL INTO THE POWER SYSTEM TOOLBOX OF THE MATLAB FOR TRANSIENT STABILITY STUDY
}

\author{
Yixin Ni \\ Dept. of EEE, The Univ. of Hong Kong \\ Hong Kong SAR
}

\begin{abstract}
In this paper, power frequency model for unified power flow controller (UPFC) is presented with its dc link capacitor dynamics included. A novel interface of UPFC to ac network for transient stability study is then suggested and realised using Power System Toolbox (PST) of the MATLAB. The control strategies for UPFC shunt and series elements are also discussed. Computer results on a 4-generator interconnected test power system show that the convergence and accuracy of the suggested interface are challenging and the suggested interface makes it very easy to incorporate UPFC model into the conventional transient stability programme. The results also show that the UPFC control strategy had strong impacts on the performance of UPFC.
\end{abstract}

\section{INTRODUCTION}

The unified power flow controller (UPFC) [1] is a member of the FACTS family with very attractive features. The development of the first commercial UPFC is being carried out under joint sponsorship of the EPRI and the WAPA of USA [2]. Study via digital simulation is conducted on electromagnetic transient programs in order to get deeper insight of UPFC control performance. [3]. However in order to study power system transient and steady-state stability, a power frequency model for UPFC and a satisfying interface of UPFC to ac transmission network are required urgently. Some pioneer work has been conducted in this area. Some of the suggested UPFC models that neglected dc link capacitor transients might lead to unacceptable error, while other UPFC models are difficult to be used in large scale power system analysis or to be incorporated in conventional transient stability programme.

In recent years, MATLAB [4] has become more and more popular in all engineering fields for its flexibility and the well support from its toolboxes. Power System Toolbox (PST) [5] is one of the powerful toolboxes that oriented to power system simulation and analysis. With its open-frame feature, it is easy to add new models into the existing program and to test the performance of these new models.

In this paper, the power frequency model of UPFC is first suggested with its dc link capacitor transients included. Then a novel interface of UPFC to ac network is developed and realised on the platform of PST. The UPFC shunt and series element control is also discussed. The shunt element control, i.e. terminal voltage magnitude and phase angle control of converter 1 , is to keep constant the UPFC terminal bus voltage magnitude and the UPFC capacitor voltage respectively. The series element control, i.e. terminal voltage magnitude and phase angle control of converter 2 , is to keep constant active and reactive power flow of the UPFC-installed line or to make UPFC act as a series compensator. The supplementary control of UPFC is added to damp power oscillations along the tie lines after disturbances. The model, interface method and control strategies are tested on a 4-generator interconnected power system [6]. The computer results show that the suggested UPFC power frequency model

\author{
Zhenyu Huang Shousun Chen Baolin Zhang \\ Dept. of EE, Tsinghua University \\ Beijing, P. R. China
}

and the UPFC-network interface method work very well in the study of system dynamic behaviour. The results also show that the different control strategies of UPFC might have noticeably different effects on the power system stability performance.

\section{MATHEMATICAL MODEL}

\subsection{UPFC Mathematical Model and Its Controls}

Figure 1 shows a schematic diagram for UPFC, where $n_{1}, X_{i 1}$ and $n_{2}, X_{i 2}$ are the voltage ratios and the reactances of the shunt and series transformers respectively. $m_{1}$ and $m_{2}$ are the voltage ratios of the shunt and series converters respectively, while $\varphi_{1}$ and $\varphi_{2}$ the firing angles with $\dot{V}_{S}$ as the reference. They represent the converter control effects between the dc- and ac-side.

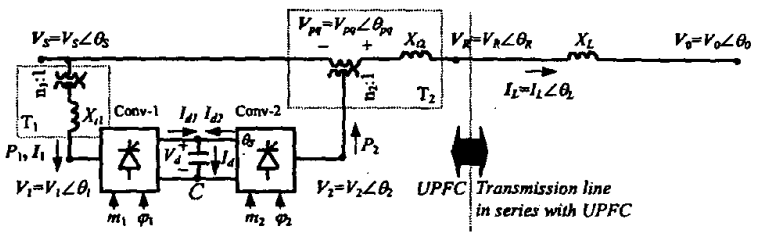

Figure 1 Transmission line with UPFC installed

The ac system uses per unit system with its variables calculated based on the system-side $S_{B}$ and $V_{B}$, while the dc variables are expressed in MKS units. With harmonics neglected, the UPFC power frequency model used in dynamic study is:

$$
\left\{\begin{array}{l}
C V_{d} \frac{d V_{d}}{d t}=\left(P_{1}-P_{2}\right) S_{B} \\
\text { where } \quad P_{1}=\operatorname{Re}\left(\dot{V}_{1}\left(\frac{n_{1} \dot{V}_{S}-\dot{V}_{1}}{j X_{t 1}}\right)^{*}\right), \quad P_{2}=\operatorname{Re}\left(\dot{V}_{p q}\left(\frac{\dot{V}_{S}+\dot{V}_{p q}-\dot{V}_{R}}{j X_{t 2}}\right)^{*}\right) \\
V_{1}=m_{1} V_{d} / V_{B} \\
\theta_{1}=\theta_{S}-\varphi_{1} \\
V_{p q}=m_{2} V_{d} / V_{B} / n_{2} \\
\theta_{p q}=\theta_{S}-\varphi_{2}
\end{array}\right.
$$

The UPFC main control includes both shunt and series element controls. The shunt element control is shown in Figures 2: (a) the constant dc link capacitor voltage control realised by controlling the firing angle $\varphi_{1}$ of converter 1 ; and (b) the constant UPFC terminal bus voltage control achieved by controlling $m_{1}$ of the PWM controller of converter 1

As for the series element, The inserting voltage $\dot{V}_{p q}$ is decomposed as $\dot{V}_{p}$, which is perpendicular to $\dot{V}_{S}$, and $\dot{V}_{q}$, in phase with $\dot{V}_{S}$ (see Figure 3). Obviously, these two components have strong impacts on real power flow and reactive power flow, respectively. Based on this decomposition, Figure 4(a) shows the constant real and reactive power flow control for the series element main control. The constant series compensation control is also shown in Figure $4(\mathrm{~b})$. Then the pairs $\left(V_{p i}, V_{q i}\right)$ will be combined into a final pair of $V_{p}$ and $V_{q}$ through weighting factors 
(see Figure $4(\mathrm{c})$ ). After passing through a smooth block the final pair $\left(V_{p}, V_{q}\right)$ is converted into corresponding $m_{2}$ and $\varphi_{2}$ for PWM control and firing angle control of inverter 2. In Figure $4(b)$, $\varphi_{2}^{\prime}$ ref $=\pi / 2+\theta_{L}-\theta_{S}$ and $V_{\text {pqref }}=K_{\text {ref }} X_{L} X_{L}$ are calculated according to the real time measurements of $\left(\theta_{L}-\theta_{S}\right)$ and $I_{L} . K_{\text {ref }}$ is the desired degree of series compensation of the tie line.

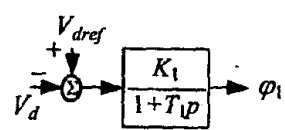

(a)

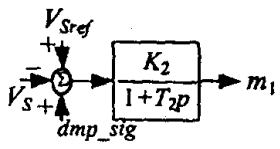

(b)

Figure 2 The shunt-element main control of UPFC

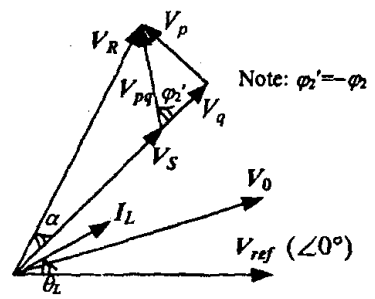

Figure 3 The phasor diagram of UPFC series element

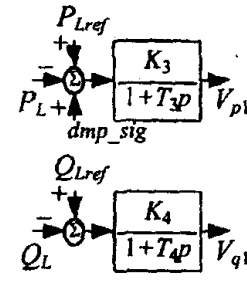

(a)

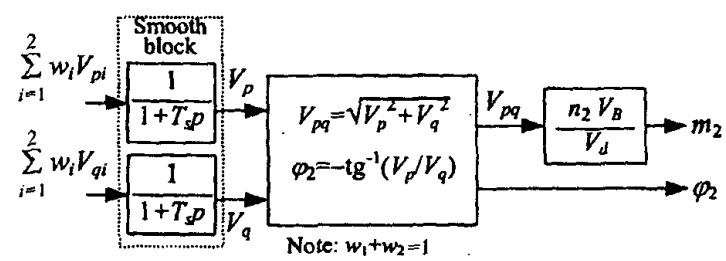

(c)

Figure 4 The series-element main control of UPFC

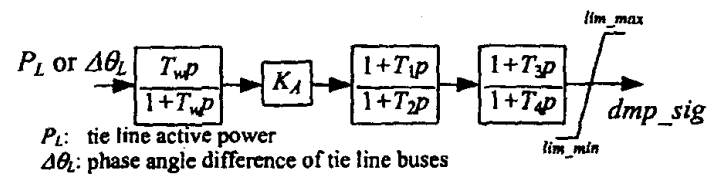

Figure 5 Supplementary control block diagram

In order to improve the damping, the PSS-like supplementary control (see Figure 5) is added to modulate the terminal bus voltage, the line real power or the series compensation degree, seen as $d m p$ sig in Figure 2(b), Figure 4(a) and (b), respectively.

\subsection{Mathematical Models for Other Power System Elements}

The mathematical models for other power system elements are as follows. The subtransient model is used for the generators with simplified third-order excitation control [7]. The mechanical power of each generator is taken as a constant. Loads are expressed as constant impedance and the ac network is linear. In order to simplify the software development, we assume $X_{d}{ }^{\prime \prime}=X_{q}{ }^{\prime \prime}=X^{\prime \prime}$ for generators so that $X^{\prime \prime}$ can be included in the bus admittance matrix, and the latter can be reduced to generator internal buses [7] and used for ac network interface to UPFC which will be discussed in detail below.

\subsection{Interface of UPFC to the ac Network}

The interface calculation of UPFC to ac network will have significant impacts on transient stability analysis speed and accuracy. The sequential solution method, which has been successfully used for ac-dc interface in ac/dc power system load flow and transient stability analysis, has been extended here for UPFC interface to ac network (see Figure 6).

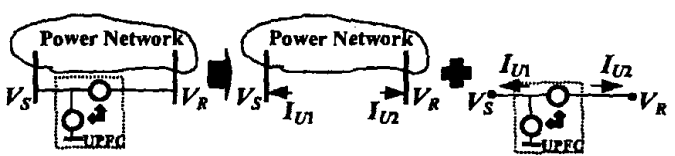

(a)

(b)

(c)

Figure 6 The interface of the UPFC to the network

(a) The integrated system

(b) AC network with FACTS modelled as 'injecting current'

(c) The 'isolated' FACTS with bus voltages from ac network

In the interface calculation we assume that the bus admittance matrix has been reduced to generator internal buses with UPFC ac terminal buses remained. The corresponding reduced bus admittance matrix equation takes the form:

$$
\left[\begin{array}{ll}
Y_{G G} & Y_{G U} \\
Y_{U G} & Y_{U U}
\end{array}\right]\left[\begin{array}{c}
\dot{E}_{G} \\
\dot{V}_{U}
\end{array}\right]=\left[\begin{array}{c}
\dot{I}_{G} \\
\dot{I}_{U}
\end{array}\right]
$$

where $\dot{E}_{G}$ : generator internal voltage behind the subtransient reactance $X^{\prime \prime} . \dot{V}_{U}=\left[\dot{V}_{S}, \dot{V}_{R}\right]^{\prime}:$ ac terminal bus voltages of the UPFC (see Figure 6). $i_{G}$ : generator currents injecting to the network. $\dot{I}_{U}=\left[\dot{I}_{U 1}, \dot{I}_{U 2}\right]^{\prime}:$ UPFC currents injecting to the network (see Figure 6).

The UPFC currents injecting to the ac network can be expressed by (see Figures 1 and 6 ):

$$
\left\{\begin{array}{l}
\dot{I}_{U 1}=-\frac{n_{1} \dot{V}_{S}-\dot{V}_{1}}{j X_{t 1}} n_{1}-\frac{\dot{V}_{S}+\dot{V}_{p q}-\dot{V}_{R}}{j X_{t 2}} \\
\dot{I}_{U 2}=\frac{\dot{V}_{S}+\dot{V}_{p q}-\dot{V}_{R}}{j X_{t 2}}
\end{array}\right.
$$

Suppose all the state variables at $t=t_{n+1}$ have been predicted according to the numerical integration, and now we are going to make network solution for algebraic variables. It is clear that in equation (3), though the UPFC output voltage magnitudes $V_{1}$ and $V_{p q}$ can be known from control output and equation (1), the phase angles of $\dot{V}_{1}$ and $\dot{V}_{p q}$ are unknown since they depend on the phase angle of $\dot{V}_{S}$. The phase angle of $\dot{V}_{S}$ is unknown that should be obtained from the network solution. Therefore an iteration approach is required to obtain the network solution. 
The iteration is conducted as follows. Substituting equation (3) into equation (2), and rearranging the second equation of equation (2), we finally have:

$\left\{\begin{array}{c}\dot{I}_{1 G}+\dot{I}_{2 G}+\left(Y_{S S}+Y_{R S}+\frac{n_{1}{ }^{2}}{j X_{t 1}}\right) \dot{V}_{S}+\left(Y_{S R}+Y_{R R}\right) \dot{V}_{R}=\frac{n_{1}}{j X_{t 1}} \dot{V}_{1} \\ \dot{I}_{2 G}+\left(Y_{R S}-\frac{1}{j X_{t 2}}\right) \dot{V}_{S}+\left(Y_{R R}+\frac{1}{j X_{t 2}}\right) \dot{V}_{R}=\frac{1}{j X_{t 2}} \dot{V}_{p q}\end{array}\right.$

where $\left[\begin{array}{ll}Y_{S S} & Y_{S R} \\ Y_{R S} & Y_{R R}\end{array}\right]=Y_{U U}$, and $\left[\begin{array}{c}\dot{I}_{1 G} \\ \dot{I}_{2 G}\end{array}\right]=Y_{U G} \dot{E}_{G}$ which can be calculated according to generator state variables. If we define a constant matrix:

$$
\tilde{Y}_{U U}=\left[\begin{array}{ll}
\left(Y_{S S}+Y_{R S}+\frac{n_{1}^{2}}{j X_{t 1}}\right) & \left(Y_{S R}+Y_{R R}\right) \\
\left(Y_{R S}-\frac{1}{j X_{t 2}}\right) & \left(Y_{R R}+\frac{1}{j X_{t 2}}\right)
\end{array}\right]
$$

and a fictitious time varying current vector:

$$
\tilde{I}_{U}=\left[\begin{array}{c}
\frac{n_{1}}{j X_{t 1}} \dot{V}_{1}-\left(\dot{I}_{1 G}+\dot{I}_{2 G}\right) \\
\frac{1}{j X_{t 2}} \dot{V}_{p q}-\dot{I}_{2 G}
\end{array}\right]
$$

we have:

$$
\widetilde{Y}_{U U} \dot{V}_{U}=\widetilde{I}_{U}
$$

The equations (5) to (7) are used for the iteration of UPFCnetwork interface as follows:

Step 1: Estimate the initial voltages of $\dot{V}_{S}{ }^{(0)}$ and $\dot{V}_{R}{ }^{(0)}$ (e. g. according to the last step), and calculate $\tilde{I}_{U}$ based on equations (6) and (1).

Step 2: Solve equation (7) for $\dot{V}_{U}=\left(\dot{V}_{S}^{(1)}, \dot{V}_{R}^{(1)}\right)^{\prime}$. If the difference of $\left(\dot{V}_{S}^{(1)}, \dot{V}_{R}^{(1)}\right)$ and $\left(\dot{V}_{S}^{(0)}, \dot{V}_{R}^{(0)}\right)$ is less than the given tolerance, then $\dot{V}_{S}^{(1)}$ and $\dot{V}_{R}(1)$ are considered as the solution of equation (4). Otherwise go to step 3

Step 3: Update $\left(\dot{V}_{S}{ }^{(0)}, \dot{V}_{R}^{(0)}\right)$ by $\left(\dot{V}_{S}^{(1)}, \dot{V}_{R}{ }^{(1)}\right)$ and repeat steps 1 and 2 till convergence is reached.

Step 1 corresponds to the UPFC calculation with estimated terminal voltages (see Figure 6(c)). Step 2 corresponds to ac network solution with UPFC effects represented as injection currents to ac network (see Figure 6(b)) except that we defined a fictitious current vector as the UPFC-network interface variable to save CPU time. After the solution of interface voltages $\dot{V}_{U}=\left(\dot{V}_{S}, \dot{V}_{R}\right)^{t}$, the first part in matrix equation (2) is used for calculation of generator injection current $\dot{I}_{G}$.

By this iteration approach there is no need to rewrite the entire program. The PST has provided the basic subroutines for machine models, reduced admittance matrix calculation, numerical integration methods, etc. So it is convenient to realize the stability analysis of the power system with UPFC simply by inserting just a few new subroutines corresponding to the UPFC model and the interface iteration into the existing software. The network solution accuracy can be controlled via the interface tolerance setting. This interface can also be extended and used for other FACTS devices.

\section{COMPUTER TEST RESULTS}

\subsection{Test System}

A 4-generator 2-area interconnected power system [6] is used for the computer test (see Figure 7). At the steady state, the electric power output of generators 1 to 4 is $700 \mathrm{MW}, 700 \mathrm{MW}, 716$ $\mathrm{MW}$, and $700 \mathrm{MW}$ respectively. The loads on the buses 3 and 13 are $967 \mathrm{MW}$ and $1767 \mathrm{MW}$. About $390 \mathrm{MW}$ power is transferred from area 1 to area 2 with a UPFC installed on one of the tie lines. The disturbance used is a three-phase stub fault on bus 3 at $t=0.50$ second, and cleared in 0.10 second. The parameters of UPFC and its control system are presented in the appendix.

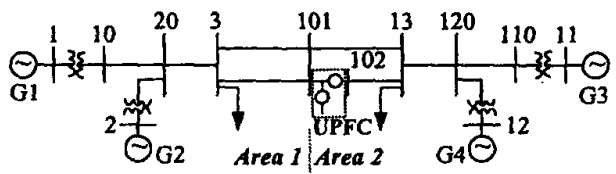

Figure 7 The computer test system diagram

\subsection{Performance of UPFC main controls}

Keeping the shunt-element control as illustrated in Figure 2, the control performances are compared with different series-element controls, i.e. the constant tie line power flow control or the constant series compensation control (see Figure 4), which are denoted as Case 1 and Case 2 respectively. The computer test results are presented in Figures 8 and 9 respectively. The figures include (a) rotor angles, (b) voltages of the fault bus 3 and UPFC sending-end bus 101, and (c) UPFC-installed tie line real power. The following observations can be made:

- The time simulation shows very good convergence in UPFC-network interface which proves that the suggested UPFC model and interface method work very well in transient stability analysis.

- With the constant power flow control, the first swing peak angle (Figure $8(\mathrm{a})$ ) is larger than that with constant series compensation control (Figure 9(a)). This means the constant series compensation has better effect on system first swing transient than the constant power flow control.

- After first swing, the constant power flow control can recover the controlled tie line power to the setting point very quickly (Figure $8(\mathrm{c})$ ) which is extremely desirable. However the constant series compensation control case will have long period of power oscillations on the controlled tie line (Figure 9(c)).

- In both cases, the terminal voltage oscillations are limited efficiently, which shows the desired control effects by the shunt-element control.

- The rotor angle plots in Figures 8 and 9 also show that system damping is very poor and supplementary damping control is necessary.

\subsection{Effects of UPFC Supplementary Control}

In order to improve the system dynamic behaviour, with the input as the real power of line 101-13, two types of supplementary control are tested, i.e., terminal voltage modulation and real power modulation, shown in Figure $2(\mathrm{~b})$ and Figure 4(a) respectively. The curves are presented in Figure 10 , including rotor angles and outputs of supplementary control. The computer results show: 
For both types, the system damping is greatly improved (see Figures 10(a) for terminal voltage modulation and Figure $10(\mathrm{c})$ for real power modulation), which means the input signal, transfer function, parameters of supplementary control are well selected and the control is effective.

The supplementary signals $d m p_{\text {_ }}$ sig of both types are similar to each other (see Figures $10(\mathrm{~b})$ for terminal voltage modulation and Figure 10(d) for real power modulation). The supplementary control output limits used are $0.2 \mathrm{p}$. u. for voltage modulation and $0.5 \mathrm{p}$. u. for power modulation respectively.

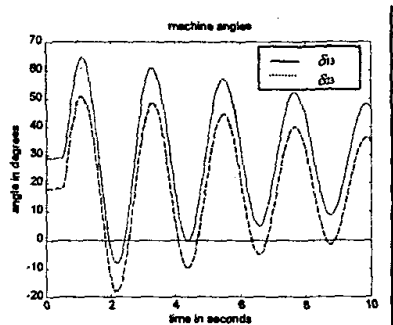

(a)

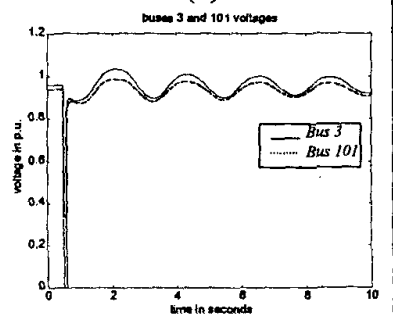

(b)

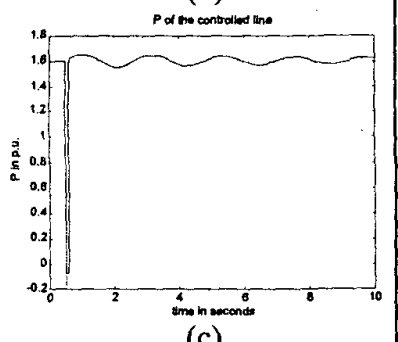

(c)

Figure 8 Plots of Case 1

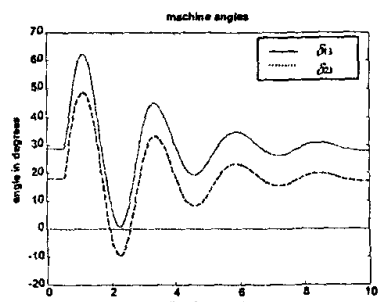

(a)

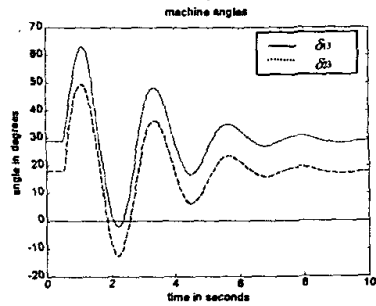

(c)

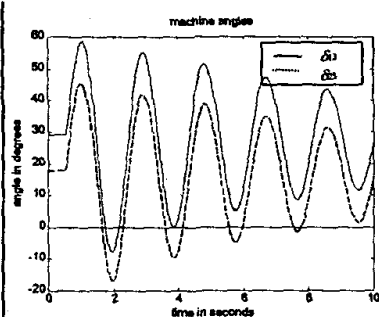

(a)

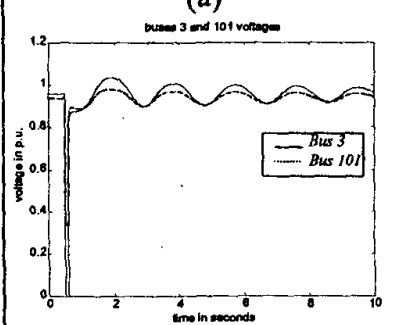

(b)

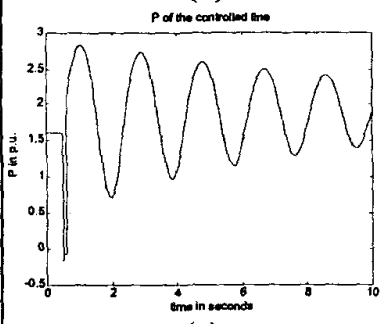

(c)

Figure 9 Plots of Case 2

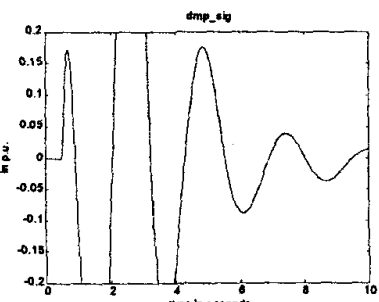

(b)

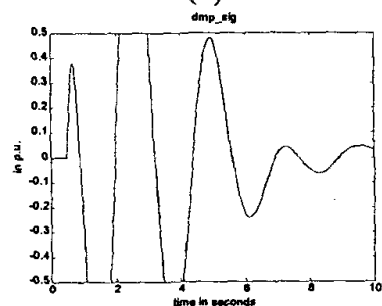

(d)
Figure 10 Effects of supplementary control

\section{CONCLUSION}

The open-frame feature of the PST and its supporting functions make it possible to easily incorporate new models into the existing transient and steady-state stability programme of PST and to test the performance of these new models. The suggested UPFC power frequency model and the newly developed UPFCnetwork interface have been realised on the platform of the PST and work very well in the study of transient stability with satisfied convergence and accuracy. The computer test results show that the constant power flow control is good for steady state control and the constant series compensation control is useful for first swing stability and the supplementary control is very efficient in damping inter-area power oscillation.

\section{ACKNOWLEDGEMENT}

The research is jointly sponsored by CRCG grant, the University of Hong Kong and Climbing B Project, State Sc. and Tech. Commission, P. R. China, to whom the authors would like to express their sincere acknowledgement.

\section{REFERENCES}

[1] L. Gyugyi, "Unified power-flow control concept for flexible ac transmission systems", IEE Proceedings-C, Vol. 139, No.4, pp:323-331, July 1992.

[2] A. S. Mehraban and A. J. F. Keri, et al., "Application of the world's first UPFC on the AEP system", EPRI-the Future of Power delivery Conference, Washington DC, 1996

[3] M. P. Bottino and B. Delfino, et al., "FACTS for more effectlve networks: unified power flow controller using PSCAD/EMTDC code", EPRI-the Future of Power delivery Conference, Washington DC, 1996

[4] A. Cavallo and R. Setola, et al., Using MATLAB, SIMULINK and Control System Toolbox, Prentice Hall Europe, 1996

[5] J. H. Chow, Power System Toolbox: A Set of Coordinated Mfiles for Use with MATLAB, Cherry Tree Scientific Software, 1996

[6] P. Kundur, Power System Stability and Control, McGrawHill Inc., 1994, pp:813-816

[7] P. Anderson and A. A. Fouad, Power System Control and Stability, IEEE Press, 1994.

\section{APPENDIX}

The parameters of UPFC:

$V_{B}=220 \mathrm{kV}, S_{B}=100 \mathrm{MVA}, n_{1}=0.05, n_{2}=0.25, X_{t 1}=0.00025$, $X_{2}=0.05$ (all in p. u. of $220 \mathrm{kV}$ base).

The parameters of UPFC main control:

$K_{1}=0.05, T_{1}=0.01, K_{2}=1.0, T_{2}=0.05, K_{3}=5.0, T_{3}=0.1, K_{4}=5.0$, $T_{4}=0.1, T_{5}=0.02, T_{6}=0.02, K_{\text {ref }}=0.4, V_{\text {dref }} \approx 22 \mathrm{kV}$.

Table Al The supplementary control parameters (T in Sec.)

$\begin{array}{ccccccc}\text { Type } & K_{A} & T_{w} & T_{I}=T_{3} & T_{2}=T_{4} & \text { lim_max } & \text { lim_min } \\ 1 & 0.83 & 3.0 & 0.02 & 0.33 & 0.2 & -0.2 \\ 2 & 0.83 & 3.0 & 0.02 & 0.19 & 0.5 & -0.5\end{array}$

